\title{
Eliminação de ruído AWGN de sinais de espectrômetros de EPR por Transformada Discreta de Fourier, com aplicação em dosimetria das radiações
}

\author{
Fábio Pelicano B. Vieira, Joyce S. Bevilacqua \\ USP - IME - Depto de Matemática Aplicada \\ 05508-090, São Paulo, SP \\ E-mail: fpbvieira@gmail.com, joyce.bevilacqua@gmail.com
}

Orlando Rodrigues Jr.

IPEN - Gerência de Metrologia das Radiações

05508-000, São Paulo, SP

E-mail: rodrijr@ipen.br

\section{Introdução e objetivo}

\section{RESUMO}

Radiações ionizantes produzem, em certas substâncias, uma quantidade de centros paramagnéticos proporcional à dose absorvida [3]. Tal quantidade pode ser obtida por espectrometria de Ressonância Paramagnética Eletrônica (Electron Paramagnetic Ressonance - EPR) [5], sendo a técnica de interesse para a área de dosimetria das radiações.

Os espectrômetros produzem espectros brutos compostos de uma assinatura do material, relacionada à presença dos centros paramagnéticos, e de ruído, que advém de fontes diversas, como a eletrônica do equipamento e acoplamento do sistema formado por cavidade, tubo e amostra, e que pode ser eliminado por pós-processamento matemático. Neste trabalho, será desenvolvido um algoritmo para identificar automaticamente a frequência de corte e eliminar o ruído de alta frequência de sinais de EPR. Aplica-se um filtro passa-baixa na Transformada Discreta de Fourier (TDF) [1] do sinal amostrado, que em seguida é reconstruído por Transformada Inversa Discreta de Fourier (TIDF) [1]. $\mathrm{O}$ algoritmo será testado em sinais simulados com base em modelo teórico e em sinais experimentais obtidos com um espectrômetro Bruker modelo EMX operando na banda X.

\section{Modelagem matemática e algoritmo}

O espectro bruto é dado por

$$
S(t)=w(t)+r(t)
$$

em que $w(t)$ representa a assinatura do material e $r(t)$, o ruído. A Figura 1 ilustra todo o processo sobre um sinal simulado, que foi gerado considerando-se que $w$ tem a forma de derivada de Gaussiana,

$$
w(t)=\left(a \cdot e^{-\frac{(t-b)^{2}}{2 c^{2}}}\right)^{\prime}=-\frac{a(t-b)}{c^{2}} e^{-\frac{(t-b)^{2}}{2 c^{2}}},
$$

sendo $a, b, c \in \mathbb{R}, \operatorname{com} a \cdot c \neq 0$.

(a) Sinal simulado com ruído

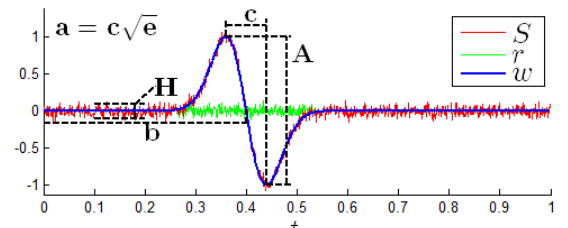

(c) Módulo da TDF filtrada

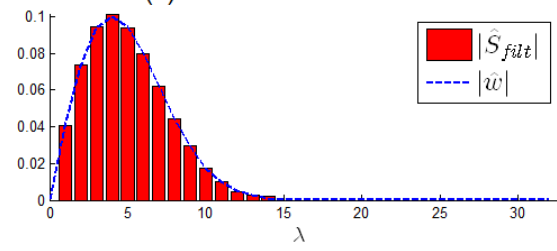

Figura 1: esquema do algoritmo (b) Módulo da TDF

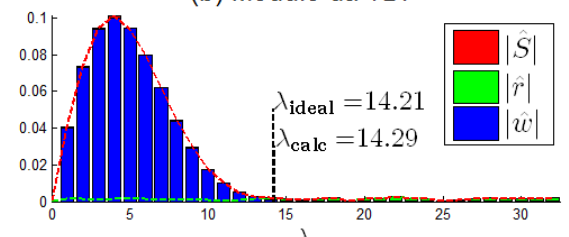

(d) Sinal reconstruîdo após a filtragem

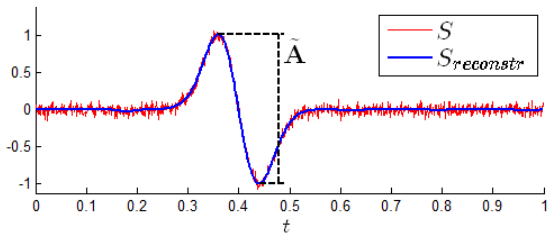


Considerou-se que o ruído $r$ é aditivo, com média nula e distribúido normalmente, com desvio-padrão $\frac{H}{4}$ e amplitude $H$. A diferença entre o máximo e o mínimo de $w$, que será denotada por $A$ e denominada amplitude do sinal objetivo, é diretamente proporcional à dose absorvida, sendo, portanto, a variável de interesse para a área de dosimetria das radiações. O algoritmo obtém, a partir de $S$, uma estimativa $\tilde{A}$ de $A$ tal que o erro relativo seja de até $0,5 \%$. A sequência de passos executados pelo algoritmo é:

1. Calcula $\hat{S}(\lambda)$ (Figura 1.b)

2. Calcula $\lambda_{\max , S}$

3. Calcula $\lambda_{\text {calc }}=3,5716 \cdot \lambda_{\max , S}$ (resultado obtido teoricamente)

4. Exclui as frequências acima de $\lambda_{\text {calc }}$ em $\hat{S}$ (Figura 1.c)

5. Calcula a TIDF de $\hat{S}$

6. O sinal reconstruído $S_{\text {reconstr }}$ é a parte real da TIDF de $\hat{S}$ (Figura 1.d)

\section{Resultados e conclusão}

Considerando-se $a=c \sqrt{e}$ e $b=0,5$, simulações foram feitas para valores de $c$ no intervalo $[0,01 ; 0,1]$ e amplitudes do ruído $H$ no intervalo $[0,02 ; 0,4]$. Para sinais simulados, os resultados foram muito bons. O erro relativo médio entre $A$ e $\tilde{A}$, para 30 execuções do algoritmo, foi inferior a $0,5 \%$ para $c$ em $[0,04 ; 0,1]$ e $H$ em $[0,02 ; 0,1]$. Como esperado, valores de $c$ no intervalo $[0,001 ; 0,03]$ produzem erros relativos médios superiores a $0,5 \%$.

Sinais experimentais produzidos pelo espectrômetro [5] a partir de uma amostra de hidroxiapatita natural irradiada, como mostra a Figura 2.a, com medidas efetuadas à temperatura ambiente, foram submetidos ao algoritmo. Nesses casos, a presença da linha de base, em geral uma componente do espectro que apresenta um período longo [2], compromete a estimativa de $\lambda_{\max }$ e faz com que $\lambda_{\text {calc }}$ (Figura 2.b) seja inferior a $\lambda_{\text {ideal }}$. Com a intervenção do usuário na estimação de $\lambda_{\text {max }}$, por simples semelhança visual com uma TDF como a exibida na Figura 1.b, o ruído de alta frequência é adequadamente eliminado, como mostra a Figura 2.c, em que $S_{\text {oper }}$ é o sinal reconstruído com a intervenção.

Conclui-se que o método é adequado para eliminar o ruído de alta frequência desde que não haja, simultaneamente, a linha de base. Isso motivou estudos adicionais sobre a remoção de rúido, em particular a linha de base [4], os quais estão em andamento.

(a) Sinal experimental

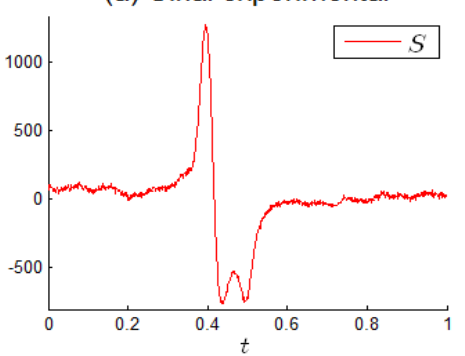

(b) Módulo da TDF

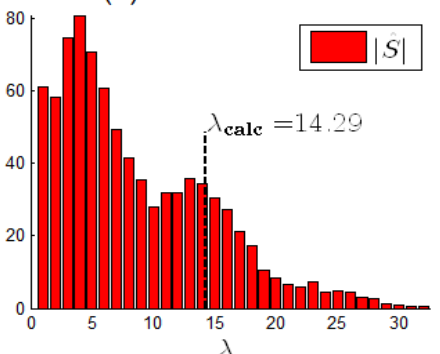

(c) Sinal reconstruído após a filtragem

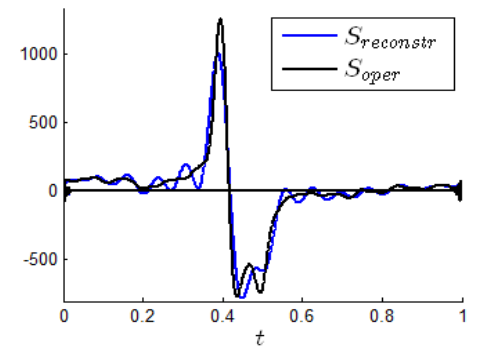

Figura 2: algoritmo aplicado sobre o espectro bruto da hidroxiapatita natural irradiada

\section{Referências}

[1] Damelin, S. B., Miller Jr., W., "The Mathematics of Signal Processing", Cambridge University Press, Cambridge, 2012.

[2] Banwell, C. N., McCash, E. M., Fundamentals of Molecular Spectroscopy, McGraw-Hill, Berkshire, 1994

[3] Desrosiers, M., Schauer, D.A., Electron paramagnetic resonance (EPR) biodosimetry, Nuclear Instruments and Methods in Physics Research B, vol. 184, pp. 219-228, (2001).

[4] Galloway, C. M., Le Ru, E. C., Etchegoin, P.G., An Iterative Algorithm for Background Removal in Spectroscopy by Wavelet Transforms, Applied Spectroscopy, Society for Applied Spectroscopy, vol. 63, núm. 12, pp 1370 - 1376, (2009).

[5] Weil, J. A., "Electron paramagnetic resonance: elementary theory and practical applications", John Wiley \& Sons, Hoboken, 2007. 